\title{
Creencias de estudiantes de pedagogía sobre la enseñanza del inglés ${ }^{1}$
}

\section{Beliefs of trainee teachers regarding English teaching}

\author{
Tania Tagle Ochoa ${ }^{2}$ \\ Claudio Díaz Larenas ${ }^{3}$ \\ Paola Alarcón Hernández ${ }^{4}$ \\ Marcela Quintana Lara ${ }^{5}$ \\ Lucía Ramos Leiva ${ }^{6}$
}

\section{Resumen}

Esta investigación tiene como propósito identificar las creencias sobre la enseñanza que tienen los estudiantes de último año de la carrera de pedagogía en inglés de una universidad chilena. La técnica utilizada para el proceso de recolección de información fue una entrevista semiestructurada. El procesamiento de los datos se realizó empleando el análisis de contenido, apoyado en la codificación y categorización de la información en el software de análisis cualitativo Atlas.ti. Los resultados indican que los participantes tienen creencias sobre la enseñanza enmarcadas en un enfoque tradicional y comunicativo del lenguaje.

\section{Palabras clave}

Formación inicial docente, creencias, enseñanza.

\section{Abstract}

The aim of this research project is to identify the opinions about the teaching which final year students studying to be English teachers have received during their degree at a Chilean university. The methodology used to collect information was semi-structured interviews. The processing of data was performed by means of a content analysis, where the codification and categorisation of the information was aided by the qualitative analysis software programme Atlas.ti. The results indicate that the participants' opinions about teaching are marked by a traditional and communicative focus of the language.

\section{Key words}

Initial teacher training, beliefs, teaching

Artículo recibido el 28 de junio de 2013 y aprobado el 7 de febrero de 2014

1 Este artículo es parte del proyecto fondecyt 1120247 titulado Investigación del conocimiento profesional, las creencias implícitas y el desempeño en aula de estudiantes de pedagogía en inglés como estrategia de generación de indicadores de monitoreo de su proceso formativo.

2 Universidad Católica de Temuco, Temuco, Chile, ttagle@uct.cl

3 Universidad de Concepción, Concepción, Chile, claudiodiaz@udec.cl

4 Profesor Asistente de la Universidad de Concepción, Concepción, Chile, palarco@udec.cl

5 Universidad Arturo Prat, Iquique, Chile, marcela.quintana@unap.cl

6 Universidad Arturo Prat, Iquique, Chile, luciaanrale@gmail.com 
Universidad Pedagógica Nacional

Facultad de Humanidades

Considerando los resultados de una prueba de inglés aplicada a nivel nacional el año 2012 a estudiantes de tercer año de educación media, el Ministerio de Educación de Chile (2013) estableció que solo un $18 \%$ de ellos logra un nivel básico en función de las competencias del área de conocimiento. Los bajos resultados obtenidos se asociarían a las prácticas pedagógicas de los docentes en el área de especialidad. A su vez, estas prácticas serían informadas por las creencias que poseerían los mismos las cuales se relacionarían con un paradigma tradicionalista sobre la enseñanza y el aprendizaje (Malderez y Wedell, 2007). Este planteamiento adquiere especial relevancia en el proceso de formación de docentes en el contexto chileno ya que, como lo indica Inostroza (1996), las representaciones que subyacen a la acción de los futuros profesionales de la educación están basadas, frecuentemente, en el llamado paradigma de transmisión de conocimientos, que se caracteriza por concebir el conocimiento como información, sustentado en verdades absolutas, ahistóricas y construido desde una lógica formal y abstracta; desligado, por lo tanto, de las experiencias del diario vivir del estudiante.

Se ha señalado que las creencias son muy difíciles de modificar por el carácter implícito de las mismas; sin embargo, algunos especialistas sugieren que su cambio es posible si se generan las instancias adecuadas para su reestructuración. Al respecto se ha planteado que la práctica, como actividad curricular, tendría un rol importante en el proceso de modificación de las creencias de los estudiantes de pedagogía en la medida que genera instancias para la acción profesional y para la reflexión de esa acción (Blázquez y Tagle, 2010).

El objetivo de esta investigación es develar las creencias que tienen los estudiantes de la carrera de pedagogía en inglés que están finalizando su preparación profesional universitaria con respecto a la enseñanza de la lengua extranjera. Estos estudiantes han participado durante cuatro, de los cinco años que tiene el plan de estudios de la carrera, en experiencias prácticas en los centros educacionales de educación básica y educación media de la región.
Al implementar sus intervenciones en el sistema educativo, ellos reflexionan sobre su desempeño profesional apoyados por los docentes a cargo de las asignaturas asociadas a la línea de práctica y didáctica de la especialidad.

\section{Marco teórico}

De acuerdo con Wallace (2002), cuando los estudiantes ingresan a un programa de formación docente ya poseen un conjunto bien establecido de representaciones sobre educación. Estas representaciones desempeñarían un rol clave en la forma en la cual ellos interpretan el proceso de enseñanza y los diferentes elementos que están involucrados en él. Algunos autores identifican este conjunto de representaciones sobre educación como constructos mentales o esquemas conceptuales. Otros identifican este conjunto de preconcepciones e ideas sobre educación como creencias (ver, por ejemplo, Joram y Gabriele, 1998).

\section{Creencias}

Borg (2006) señala que la importancia de las creencias ha sido estudiada en muchas áreas de interés para los profesionales expertos en la enseñanza del inglés; por ejemplo, se ha investigado sobre la influencia de las creencias pedagógicas en las conductas de clase de los docentes y en los programas de formación inicial o continua del profesorado.

Díaz y Solar (2011) sostienen que las creencias pueden ser entendidas como redes complejas de procesamiento alojadas en la memoria de largo plazo de los docentes. Considerando esta perspectiva, las creencias actúan como filtros del conocimiento teórico adquirido, redefiniendo y transformando el mismo en conocimiento práctico, personal y subjetivo, el cual permite que los profesionales de la educación entiendan el mundo y actúen según él. En el mismo marco, los autores anteriormente mencionados sostienen que las creencias intervienen en la conducta y forma de enseñar de los docentes, influyendo también en sus decisiones y modos de desempeñarse dentro del aula. 
Farrell (2009) plantea que las creencias se encuentran alojadas en sistemas que las conectan y relacionan entre sí. De este modo, las creencias más antiguas y que se encuentran en lo más profundo de estos sistemas son las más difíciles de modificar. Es relevante señalar que las creencias se derivan de experiencias en contextos educaciones formales, relacionadas con los años de escolaridad, e informales, las cuales involucran interacción con los miembros del entorno (Richards y Lockhart, 2007; Díaz y Solar, 2009). Al respecto, Mak (2011) sugiere que las creencias más difíciles de modificar son aquellas adquiridas en contextos educacionales formales.

La idea de que las creencias de los estudiantes sobre la enseñanza y el aprendizaje se desarrollan observando a sus propios profesores desempeñando su rol profesional ha sido discutida por diferentes investigadores (ver, por ejemplo, Levin y He, 2008). Se ha argumentado que estas representaciones son extremadamente difíciles de cambiar y afectan la manera en que los estudiantes interpretan nuevas ideas o información sobre el aprendizaje y la enseñanza, y en cómo esas ideas son trasladadas a la práctica.

Asociado a lo anterior, Wright (2010) indica que los estudiantes de pedagogía que ingresan a programas de formación inicial usualmente lo hacen con una visión de la enseñanza simple y ligada a la transmisión. Considerando esta perspectiva, las creencias con respecto al proceso de enseñanza y aprendizaje se mantendrían en el transcurso de programas de formación docente, prolongándose en las prácticas pedagógicas.

Algunos autores enfatizan que la modificación de las creencias corresponde a un proceso complejo. Cuando los docentes intentan cambiar su sistema de creencias y prácticas ocurre un desequilibrio entre las mismas. Los docentes experimentan sentimientos de incomodidad y frustración a medida que intentan alinear sus prácticas pedagógicas con un sistema de creencias en expansión o alterado. En este sentido, los docentes tienden a dejar de lado la información que es opuesta a sus creencias (Díaz, Martínez, Roa y Sanhueza, 2010a).
Creencias de estudiantes de pedagogía sobre la enseñanza del inglés Tania Tagle Ochoa, Claudio Díaz Larenas, Paola Alarcón Hernández,
Marcela Quintana Lara, Lucía Ramos Leiva

Un factor que parece afectar el desarrollo o cambio de las creencias previas de los estudiantes durante el proceso de formación profesional dice relación con el carácter implícito de estas. En este marco, se ha planteado que las creencias usualmente permanecen a un nivel tácito y, por lo tanto, son difíciles de articular y explorar; este hecho, a su vez, explicaría la perpetuación de las prácticas pedagógicas tradicionales (Pajares, 1992; Levin y Wadmany, 2006).

Considerando la modificación de las creencias pedagógicas, Blázquez y Tagle (2010) indican que las mismas pueden ser transformadas por medio de la experiencia. Estos autores sugieren que la reflexión sobre la práctica pedagógica es importante en el proceso de modificación de las creencias. Distintos autores establecen que las creencias pueden ser transformadas por medio de la reflexión (ver, por ejemplo, Richards y Farrell, 2005; Prieto, 2008). En este sentido, Leshem y Bar-Hama (2008) sugieren que los docentes pueden modificar sus creencias al establecer conexiones entre antecedentes teóricos y prácticos relacionados con el propio desempeño profesional.

\section{Enseñanza}

Malderez y Bodòczky (2002) señalan que la enseñanza es una competencia compleja y abierta. Es compleja porque los docentes realizan o desarrollan muchos procesos al mismo tiempo con diferentes personas en diferentes contextos y es abierta porque no hay una forma correcta de realizar un determinado proceso, en otras palabras, hay diferentes formas de aproximarse y de satisfacer las necesidades de aprendizaje de las personas y existe una variedad de cursos de acción para satisfacer demandas similares. Williams y Burden (2001) plantean que, desde una perspectiva constructivista, no hay una determinada forma de enseñar ya que la preocupación más importante de esta práctica social es desarrollar el aprendizaje. Relativo a lo anterior, Malderez y Bodòczky (2002) señalan que el aprendizaje es el objetivo de la enseñanza y este, en términos de desa- 
Universidad Pedagógica Nacional

Facultad de Humanidades

rrollo de constructos, es esencialmente un proceso distinto para cada individuo.

En este contexto, Díaz, Martínez, Roa y Sanhueza (2010b) manifiestan que la enseñanza del idioma inglés se caracteriza por ser un proceso complejo para los docentes debido a que las variables contextuales e institucionales, y la realidad socio-económica de los estudiantes pueden convertirse en agentes mediadores y/o obstaculizadores del aprendizaje de este lenguaje.

\section{Descripción del contexto}

El presente estudio fue realizado en una universidad ubicada en el sur de Chile, específicamente, en la Facultad de Educación, unidad académica a la que pertenece la carrera de pedagogía en inglés. La competencia profesional de los estudiantes de esta carrera se desarrolla a través de distintas experiencias de aprendizaje incluidas en los cursos del plan de estudio, principalmente, a través de las realizadas en los cursos llamados Talleres Pedagógicos (línea de práctica progresiva) y en la práctica profesional o final. Las actividades asociadas a los Talleres Pedagógicos se focalizan en que los estudiantes, entre otros aspectos, expliciten y cuestionen las creencias sobre el proceso de enseñanza aprendizaje que orientan sus prácticas reflexionando sobre su desempeño profesional.

\section{Metodología}

\section{Diseño de la investigación}

El diseño de esta investigación es de carácter cualitativo. Al respecto, Sandín (2003) sugiere que el estudio de las creencias sería un dominio de investigación asociado, en esencia, al paradigma cualitativo por el carácter de las mismas y por estar profundamente relacionadas con el pensamiento. Con respecto a las características de un diseño de investigación de carácter cualitativo, Galeano (2004) señala que este es abierto en función de la selección de los participantes y las formas en que se analizará la información.

\section{Informantes}

Respecto a los sujetos de la investigación, es relevante mencionar que los mismos fueron considerados para participar en un estudio de casos (Stake, 1998). Al respecto, se seleccionaron, al azar, diez estudiantes que estaban finalizando su proceso de formación profesional en la carrera de pedagogía en inglés, es decir, alumnos que estaban realizando su práctica profesional en establecimientos de la región. Algunos de los criterios de exclusión de los participantes se asociaron a que los mismos no hubiesen interrumpido sus estudios en la universidad por compromisos de tipo laboral, ni hubiesen formado parte de programas de movilidad estudiantil en países de habla inglesa. Lo anterior con el objetivo de que sus creencias no se vean influenciadas por experiencias profesionales y/o formativas desarrolladas en forma externa al proceso de preparación en la institución de educación superior.

\section{Técnica de generación de los datos}

El instrumento utilizado para recopilar información fue una entrevista semiestructurada (ver protocolo de entrevista en anexo 1). Con respecto a las entrevistas de carácter semiestructurado, Bisquerra (2009) sostiene que las mismas deben partir desde un esquema o pauta establecida, teniendo en consideración, de forma anticipada, los datos que se necesitan recolectar.

\section{Procedimiento}

En cuanto al procedimiento seguido, es importante mencionar que se elaboró un protocolo de entrevista validado a través del juicio de expertos. Se desarrolló la entrevista a cada participante. Cada entrevista se prolongó por, aproximadamente, 40 minutos. El procesamiento de los datos se realizó empleando el análisis de contenido, apoyado en la codificación y categorización de la información en el software de análisis cualitativo Atlas.ti. 


\section{Resultados}

\section{Análisis de resultados}

La categoría creencias sobre la enseñanza del inglés, presentada en la red conceptual, permite identificar las siguientes subcategorías: transmisión de información y desarrollo de habilidades.

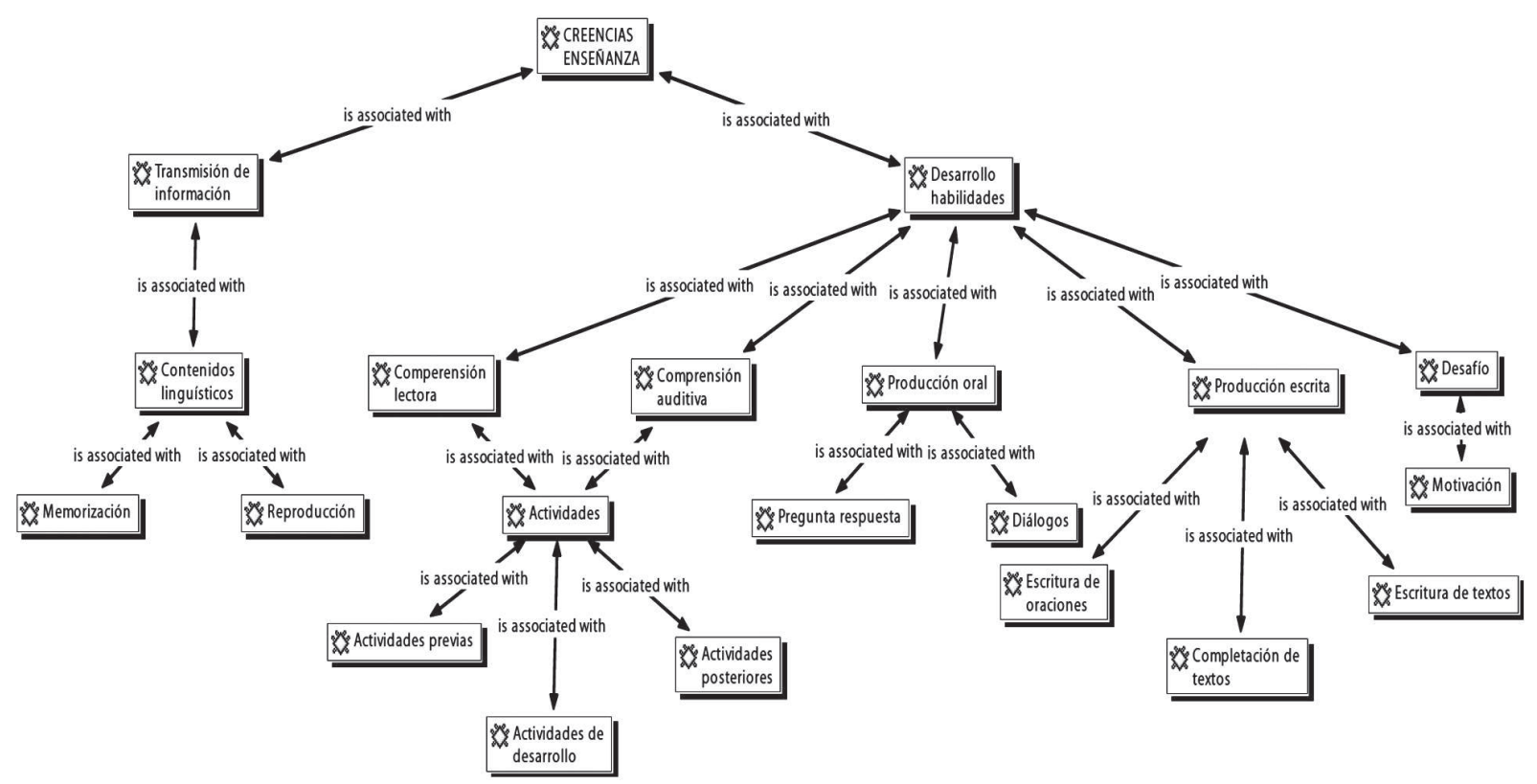

En el marco de la red conceptual, la cual hace referencia a creencias sobre la enseñanza del inglés, se presenta como primera subcategoría transmisión de información. Respecto a esto, cuando a los estudiantes se les pregunta por lo que ellos entienden por este proceso, uno de ellos indica lo siguiente: "Enseñar es entregarle información a los alumnos..." (E10 [21:21]). Otro estudiante plantea que "enseñar es pasar materia...” (E01 [27:27]). Relativo a lo anterior, algunos estudiantes de la carrera parecen representarse este proceso como uno asociado a la entrega de información, esencialmente, de contenidos lingüísticos. Relacionado con esto, un estudiante afirma:

No sé si lo más importante para todos (se refiere a los estudiantes de la carrera de pedagogía en inglés) es la gramática o no, pero por lo menos para mí sí lo es, porque si yo quiero enseñar inglés a un alumno, primero, tiene que manejar la estructura gramatical para producir oraciones. Después, tendríamos ejercicios que ayuden a ampliar el vocabulario de los estudiantes. El vocabulario es una parte muy importante de la enseñanza del inglés. (E07 [36:36])

En este mismo contexto, otro estudiante plantea:

Para mí lo fundamental es la gramática y el vocabulario porque si los alumnos quieren entender cualquier texto, ya sea oral o escrito, primero tienen que dominar un poco de léxico en inglés para ir entendiendo qué significa lo que leen o escuchan; por otro lado, si se está hablando en presente, pasado o futuro... entonces, para mí, esos dos aspectos son fundamentales para el desarrollo del inglés, la gramática y el vocabulario. (E06 [50:50])

Los contenidos lingüísticos anteriormente identificados serían aprendidos a través de procesos de memorización y reproducción. Respecto a esto, un estudiante señala lo siguiente: "También tengo que hacer que mis alumnos sean capaces de producir 
Universidad Pedagógica Nacional

Facultad de Humanidades

todo lo que ellos han memorizado, todo lo que han logrado internalizar, ya sea de gramática, de léxico, etcétera..." (E04 [36:36]).

En síntesis, en relación a las respuestas dadas por los estudiantes, se puede identificar que algunos de ellos parecieran tener creencias asociadas a una propuesta tradicional de la enseñanza del lenguaje, al menos, a nivel de conocimiento declarativo. La propuesta tradicional se caracterizaría por concebir la enseñanza como un proceso de transmisión de información. La información trasmitida se asociaría a los contenidos de tipo lingüístico (por ejemplo, gramática y el vocabulario) los cuales se aprenderían a través de la memorización y reproducción de estos. Este tipo de creencias tenderían a "cosificar" la naturaleza del lenguaje omitiéndose la esencia comunicativa del mismo.

La segunda subcategoría que se presenta en la red conceptual es desarrollo de habilidades. Algunos de los estudiantes entrevistados relacionan la enseñanza del idioma con las cuatro habilidades lingüísticas (producción y comprensión oral/escrita). Respecto a esto, un estudiante indica que se enseña inglés cuando se "desarrollan las cuatro habilidades las que corresponden a hablar, escribir, leer y escuchar... una habilidad del lenguaje se encuentra relacionada con la otra, tenemos que leer y escuchar, pero también escribir y leer para poder comunicarnos" (E03 [10:10]). En este mismo marco, algunos estudiantes sugieren que la comprensión lectora y la comprensión auditiva se desarrollan a través de actividades previas, de desarrollo y posteriores a la lectura o audición. Las actividades previas a la lectura/ audición se utilizarían para que los alumnos activen conocimientos previos, se planteen un propósito para lectura/audición, y se preparen para dificultades lingüísticas. Un estudiante indica lo siguiente: "En las actividades previas, trato de introducir las palabras que van a ser incluidas en una canción o en un cuento" (E05 [49:49]). Otro participante enfatiza la predicción de información en textos orales o escritos en función de las actividades previas y afirma: "En las actividades previas, uno siempre hace a los alumnos predecir el contenido del texto a través de presentación de imágenes y videos, lectura del título del texto o haciendo preguntas sobre la temática” (E08 [58:58]).

En el marco de las actividades de desarrollo, las mismas se focalizarían en identificar información general y específica de los textos seleccionados, proceso que se llevaría a cabo por medio de diferentes tareas. Un participante manifiesta que "en las actividades durante la lectura, los alumnos leen e identifican las ideas principales y secundarias del texto a través de preguntas, tareas de selección múltiple, ejercicios de verdadero y falso" (E02 [53:53]). En el contexto de las actividades posteriores, las mismas se centrarían en constatar la comprensión de lo leído/ escuchado por parte de los alumnos. Al respecto, un participante señala que: "Los alumnos finalizan la actividad de lectura escribiendo sobre lo que ellos entendieron del texto" (E10 [51:51]).

Considerando las respuestas dadas por los participantes, relativas al desarrollo de las habilidades receptivas, se pueden identificar creencias respecto a la enseñanza del lenguaje enmarcadas en una visión de tipo comunicativa. La visión comunicativa se distinguiría por representarse la enseñanza asociada a la generación de contextos significativos de aprendizaje en los que se activarían conocimientos previos, se realizarían predicciones con respecto al contenido basándose en elementos textuales, y se identificaría información general y específica en función del propósito de la lectura o de la audición.

Considerando la subcategoría previamente presentada, en el marco del desarrollo de la producción oral, algunos de los docentes en formación asocian la misma a actividades de pregunta-respuesta. Con respecto a lo anterior, uno de los participantes señala lo siguiente: "Yo trabajo con diálogos cortos, en este caso, dependiendo del nivel, pregunta y respuesta" (E10 [44:44]). Relacionado con lo expuesto previamente, otro entrevistado indica: "Yo les hago preguntas en inglés y ellos me dan respuestas inmediatas, no copiadas de las guías, sino que ellos las arman solitos" (E07 [57:57]). Por otro lado, algunos de los entrevistados también relacionan los diálogos con la producción oral del idioma 
inglés. Con respecto a lo anterior, un participante plantea que: "Para enseñar a hablar en inglés, ellos (se refiere a los alumnos) deben crear sus propios diálogos tomando en consideración ciertas estructuras gramaticales" (E05 [39:39]). Otro entrevistado destaca la repetición y corrección de diálogos en una clase de inglés: "Cuando utilizo diálogos, yo los leo y hago que los alumnos los repitan después de mí. Al mismo tiempo, los voy corrigiendo si es que cometen errores" (E06 [33:33]).

Considerando la subcategoría de desarrollo de habilidades, los docentes en formación también asocian la misma a la producción escrita. Este proceso se llevaría a cabo por medio de la escritura de oraciones, como lo señala un participante en el siguiente segmento de texto: "En instancias de producción escrita, hago que los alumnos escriban oraciones" (E01 [24:24]). Los participantes también relacionan la producción escrita con tareas que involucran la completación de textos. En este marco, un participante manifiesta:

Para enseñar a escribir en inglés, yo considero la completación. Primero que todo, les presento una carta para que ellos escriban una, pero no les doy el espacio en blanco sino que ellos deben completar las partes donde falta información. La actividad siempre se realiza con una guía porque si uno los deja solos no saben por dónde comenzar. (E04 [63:63])

Otra de las formas que predomina en el desarrollo de habilidades de producción escrita, por parte de los docentes en formación, es la escritura de textos. Relativo al punto previo, un participante señala:

Yo enseño a los alumnos a escribir posterior a la presentación de estructuras gramaticales. Primero, se enseña la estructura gramatical, después de eso se escriben ejemplos en la pizarra y, luego, los alumnos escriben. Con respecto a las actividades que selecciono, hace poco los hice hacer un ensayo. Primero les enseñé las partes del ensayo y después escribieron el mismo utilizando pasado simple. (E03 [20:20])

En función de la información expuesta previamente, las formas en que los participantes enseñan a escribir serían similares a aquellas relacionadas con la producción oral. En este sentido, la producción escrita de la lengua inglesa se desarrollaría tomando como base determinadas estructuras gramaticales. Este proceso, al igual que el relacionado con la producción oral, se llevaría a cabo por medio de tareas de repetición, rutinarias y relativamente mecánicas, lo que se asociaría con creencias tradicionales con respecto a la enseñanza de la lengua extranjera.

Finalmente, es importante mencionar que algunos participantes relacionan la enseñanza del inglés con un desafío que se les plantea desde el sistema educativo por la aparente desmotivación que presentarían los alumnos con respecto a la asignatura. Un estudiante señala: "La enseñanza del idioma inglés es desafiante, no imposible, mientras los profesores sepan adaptar las clases a sus contextos y realidades" (E09 [20:20]). En este mismo marco, otro estudiante indica: "Para mí enseñar inglés es un tremendo desafío porque no todos quieren aprender el idioma, hay un rechazo muy grande hacia la asignatura por parte de los alumnos" (E04 [25:25]). En este contexto, debido a la actitud de los alumnos del sistema educacional hacia la asignatura de inglés, los docentes en formación manifiestan que la enseñanza de la lengua inglesa debe privilegiar la motivación de los estudiantes por medio de diferentes estrategias. Lo anterior, se ve evidenciado en el siguiente segmento de entrevista:

Es necesario enseñar de forma distinta al resto de las asignaturas porque parte por un asunto de motivación. Si es que uno logra hacer el aprendizaje entretenido los alumnos, se interesan y eso provoca que ellos quieran aprender más y pongan más de su parte. (E05 [29:29])

\section{Discusión de resultados}

Se puede identificar que los participantes en esta investigación tienen creencias asociadas a una visión tradicional y también a una comunicativa de la enseñanza del lenguaje. Los estudiantes de la carrera de pedagogía en inglés poseerían creencias sobre la enseñanza del idioma asociadas a un proceso de transmisión de información, específicamente, 
Universidad Pedagógica Nacional

Facultad de Humanidades

de contenidos lingüísticos por medio de la memorización y reproducción. Desde la perspectiva de Larsen-Freeman (2008), esta práctica se basaría en una propuesta tradicional de enseñanza de la lengua inglesa en la cual el profesor es una autoridad en la sala de clases y expositor de contenidos de aprendizaje, tales como: estructuras gramaticales e ítems de vocabulario. Desde esta visión de la enseñanza, los aprendices actuarían como receptores pasivos de información (Malderez y Bodòczkzy, 2002).

Por otro lado, los participantes del estudio también poseerían creencias sobre la enseñanza del idioma asociadas al desarrollo de las habilidades lingüísticas, es decir, creencias enmarcadas en una visión que se relaciona con el uso del lenguaje en el proceso de comunicación.

Con respecto al desarrollo de la comprensión oral/escrita, los entrevistados parecen coincidir en que es conveniente utilizar diferentes tipos de actividades en la lectura/audición de distintos tipos de textos. Lo anterior se puede interpretar como una consideración hacia la naturaleza de los textos y sus características en la selección de tareas que ayuden a aproximarse hacia su significado (McDonough, Shaw y Masuhara, 2013). Los entrevistados también parecen coincidir en que las actividades previas, durante y posteriores de la lectura o de la audición son igualmente relevantes. En este contexto, los participantes, en general, estarían considerando que tanto los modos de procesamiento top-down como bottom-up son importantes en el proceso de comprensión, lo cual a su vez, puede significar que le están asignando relevancia tanto a los conocimientos lingüísticos previos como a los referidos al tópico, al tipo de texto, entre otros (Flowerdew y Miller, 2005).

Si se considera que las creencias sobre la enseñanza son difíciles de cambiar ya que se construyen tempranamente a través de las experiencias de los estudiantes en contextos formales de educación en los cuales observan a los docentes desempeñando su rol profesional y que, además, las creencias de los docentes sobre la enseñanza del inglés, en el contexto regional, están enmarcadas básicamente en una visión tradicionalista y estructural del lenguaje (ver a Blázquez y Tagle, 2010), se puede identificar que el proceso formativo, a nivel universitario, ha tenido algún impacto en la modificación de las creencias por parte de los participantes del estudio. Respecto a esto, es importante señalar que los estudiantes participantes en la investigación tienden a relevar las experiencias vividas en los cursos de didáctica y práctica (Talleres Pedagógicos) como sucesos significativos en el cambio experimentado. Para ilustrar el punto previo se adjuntan los siguientes segmentos de entrevista:

Entrevistadora: ¿Crees que la experiencia en la universidad está asociada, de alguna forma, a la importancia que le estás asignando al uso del inglés en la sala de clases?

Entrevistada 1: Sí, por supuesto porque quizás si me hubiesen dicho siga enseñando con el enfoque gramatical lo hubiera seguido haciendo... con los profesores de la especialidad y con los de didáctica siempre hemos reflexionado sobre eso...el porqué es mejor enseñar así, no quitándole importancia a lo que es la gramática, pero enfocándonos más en la enseñanza del inglés desde un enfoque comunicativo.

Entrevistadora: Si tú tuvieras que identificar algún elemento en tu proceso formativo que ha impactado en que cambiaras las ideas originales que tenías sobre la enseñanza ¿Qué elemento sería destacarías?

Entrevistada 2: Las prácticas... porque los establecimientos y los alumnos que conocí eran todos tan distintos que me obligaban a esforzarme a pensar cómo voy a lograr que estos alumnos aprendan con las características que tienen.

Por otro lado, se pueden identificar en los participantes creencias asociadas a la producción oral y escrita enmarcadas más bien en una propuesta tradicionalista del lenguaje en la cual prevalecen ideas asociadas a la ejercitación, repetición, y segmentación del mismo. Esta visión de la enseñanza se relaciona con la implementación de tareas focalizadas en la formación de hábitos (Larsen-Freeman, 
2008). Richards (2006) asocia esta representación de la enseñanza a una "práctica mecánica", la cual los aprendices pueden llevar a cabo sin necesidad de comprender lo que producen oralmente en cuanto a relaciones de significado.

En cuanto a la producción escrita, los estudiantes parecen tener una representación de esta habilidad como aquella asociada al proceso de copiar o reproducir información. Esta concepción de enseñanza es de carácter limitado debido a que solo se lleva a cabo a nivel de la oración, teniendo como foco principal el aprendizaje teórico de estructuras gramaticales e ítems de vocabulario (Hall, 2011).

Considerando lo planteado con anterioridad, parece relevante preguntarse por qué existirían representaciones asociadas a una visión comunicativa de la lengua extranjera cuando se hace referencia al desarrollo de la enseñanza de las habilidades receptivas e ideas un tanto tradicionales basadas en un paradigma de transmisión de información que alude a las habilidades productivas. Relativo a esto, y considerando que las creencias tradicionales con respecto a la enseñanza se modificarían a través de los procesos de reflexión sobre la acción, es posible inferir que los participantes tienen pocas o insuficientes instancias, a través de su progreso formativo, para implementar acciones basadas en un enfoque comunicativo o de uso de lenguaje en la enseñanza de las habilidades productivas (expresión oral y escrita). Lo anterior puede explicar la reproducción de prácticas tradicionalistas en este ámbito.

Finalmente, es importante señalar que los participantes en el estudio también perciben la enseñanza del idioma como un desafío, en el cual se buscan promover diferentes estrategias para motivar a los alumnos. En función de lo señalado previamente, distintos autores enfatizan que el rol del profesor, en el marco de esta concepción sobre la enseñanza, se relaciona con incentivar a los alumnos para que realicen actividades que involucren la comunicación (Richards y Rodgers, 2007).

\section{Conclusiones}

Considerando la información previamente presentada se puede señalar que los participantes en este estudio tienen creencias enmarcadas en una visión tradicional y también en una comunicativa de la enseñanza del lenguaje. Al respecto, y como se sugirió al inicio de este artículo, la visión tradicional que tienen los estudiantes en formación (futuros docentes) sobre la enseñanza de la lengua extranjera pudiese estar afectando los resultados de aprendizaje que obtienen los alumnos del sistema en el área de especialidad. Lo anterior porque las creencias permean el proceso de toma de decisiones a nivel de la práctica pedagógica. En este contexto, a través del proceso formativo, se requiere perfeccionar la implementación de iniciativas, asociadas a la línea de práctica, que favorezcan que los estudiantes de pedagogía en inglés puedan explicitar, cuestionar y modificar sus creencias sobre cómo se construye conocimiento en el área de especialidad. Al respecto, en la preparación profesional, se necesita poner especial atención a iniciativas que logren cuestionar las creencias asociadas a las tareas $o$ actividades que se pueden diseñar o implementar para el desarrollo de las habilidades productivas (expresión oral y escrita) las cuales tienden a basarse, esencialmente, en una visión tradicional de la enseñanza de la lengua.

\section{Referencias}

Bisquerra, R. (2009). Metodología de la investigación cualitativa [2 $2^{\mathrm{da}}$ ed.]. Madrid: Editorial la Muralla.

Borg, S. (2006). Teacher cognition and language education: Research and practice. Londres/Nueva York: Continuum.

Blázquez, F. y Tagle, T. (2010). Formación docente: Un estudio de las creencias de alumnos y profesores sobre el proceso de enseñanza y aprendizaje del inglés. Revista Iberoamericana de Educación, 54(4), $1-12$.

Díaz, C. y Solar, M. (2009). El profesor universitario: Construcción de su saber pedagógico e identidad profesional a partir de sus cogniciones y creencias. Revista Calidad en la Educación, 30, 208-232. 
Universidad Pedagógica Nacional

Facultad de Humanidades

Díaz, C. y Solar, M. (2011). La revelación de las creencias lingüístico-pedagógicas a partir del discurso del profesor de inglés universitario. Revista de Lingüística Teórica y Aplicada, 49(2), 57-86.

Díaz, C., Martínez, P., Roa, I. y Sanhueza, M. (2010a). Los docentes en la sociedad actual: Sus creencias y cogniciones pedagógicas respecto al proceso didáctico. Revista de la Universidad Bolivariana, 9(25), 421-436.

Díaz, C., Martínez, P., Roa, I. y Sanhueza, M. (2010b). Una fotografía de las cogniciones de un grupo de docentes de inglés de secundaria acerca de la enseñanza y aprendizaje del idioma en establecimientos educacionales públicos de Chile. Revista Folios, 31, 69-79.

Farrell, T. (2009). Critical reflection in a TESL course: Mapping conceptual change. ELT Journal, 63, 221-229.

Flowerdew, J. y Miller, L. (2005). Second language listening: Theory and practice. Nueva York: Cambridge University Press.

Galeano, M. (2004). Diseño de proyectos en la investigación cualitativa. Medellín: Universidad Eafit.

Hall, G. (2011). Exploring English language teaching: Language in action. Oxon: Routledge.

Inostroza, G. (1996). Talleres pedagógicos: Alternativas en formación docente para el cambio de la práctica de aula. Santiago: Dolmen Ediciones.

Joram, E. y Gabriele, A. (1998). Preservice teachers' prior beliefs: Transforming obstacles into opportunities. Teaching and Teacher Education 14/2,175-191.

Larsen-Freeman, D. (2008). Techniques and principles in language teaching [ $2^{\mathrm{da}}$ ed.]. Oxford: Oxford University Press.

Leshem, S. y Bar-Hama, R. (2008). Evaluating teaching practice. ELT Journal, 62(3), 257-265.

Levin, B. y He, Y. (2008). Investigating the content and sources of preservice teachers' personal practical theories. Journal of Teacher Education, 59(1), 55-68.

Levin, T. y Wadmany, R. (2006). Teachers' beliefs and practices in technology-based classrooms: A developmental view. Journal of Research on Technology in Education, 39(2), 157-181

Mak, S. (2011). Tensions between conflicting beliefs of an EFL teacher in teaching practice. RELC Journal, 42(53), 53-67.

Malderez, A. y Bodòczky, C. (2002). Mentor courses: A resource book for trainer-trainers. Cambridge: Cambridge University Press.
Malderez, A. y Wedell, M. (2007). Teaching teachers: Processes and practices. Londres: Continuum.

McDonough, J., Shaw, C., y Masuhara, H. (2013). Materials and methods in ELT: A teacher's guide [ $3^{\mathrm{er}}$ ed.]. Oxford: Wiley-Blackwell.

Ministerio de Educación de Chile (2013). Resultados SIMCE III Medio 2012. Recuperado el 13 de junio, 2013 en http://www.mineduc.cl/usuarios/mineduc/ doc/201306061729100.Resultados_SIMCE_ingles_ III_Medio_2012.pdf

Pajares, F. (1992). Teachers' beliefs and educational research: Cleaning up a messy construct. Review of Educational Research, 62(3), 307-332.

Prieto, M. (2008). Creencias de los profesores sobre evaluación y efectos incidentales. Revista de Pedagogía, 29(84), 123-144.

Richards, J. (2006). Communicative language teaching today. Nueva York: Cambridge University Press.

Richards, J. y Farrell, T. (2005). Professional development for language teachers: Strategies for teacher learning. Cambridge: Cambridge University Press.

Richards, J. y Lockhart, C. (2007). Reflective teaching in second language classrooms. Nueva York: Cambridge University Press.

Richards, J. y Rodgers, T. (2007). Approaches and methods in language teaching. Cambridge: Cambridge University Press.

Sandín, M. (2003). Investigación cualitativa en educación: Fundamentos y tradiciones. Madrid: McGrawHill.

Stake, R. (1998). Investigación con estudios de casos. Madrid: Morata.

Wallace, M. (2002). Training foreign language teachers: A reflective approach. Cambridge: Cambridge University Press.

Williams, M. y Burden, R. (2001). Psychology for language teachers: A social constructivist approach. Cambridge: Cambridge University Press.

Wright, T. (2010). Second language teacher education: Review of recent research on practice. Language Teaching, 43, 259-296. 


\section{Anexo 1: Guion para entrevista}

semi-estructurada [T2]

- ¿Qué significa para usted enseñar?

- ¿Cómo se enseña inglés?

- ¿Qué objetivos son relevantes para lograr en una clase de inglés?

- ¿Qué contenidos son importantes de cubrir en una clase de inglés?

- ¿Cómo enseña a hablar en inglés?

- ¿Cómo enseña a escribir en inglés?

- ¿Cómo se enseña a comprender textos hablados en inglés?

- ¿Cómo se enseña a comprender textos escritos en inglés? 